\title{
A NEW APPROACH TO DERIVING INTERATOMIC MANY-BODY INTERACTIONS IN METALS
}

\author{
Z. GURSKII ${ }^{a, b *}$ AND J. KRAWCZYK ${ }^{b \dagger}$ \\ anstitute for Condensed Matter Physics, Ukrainian Academy of Sciences \\ vul. Svientsitskogo 1, 290011; Lviv, Ukraine \\ ${ }^{b}$ Opole University, Oleska 48, 45-052 Opole, Poland
}

(Received January 29, 1998; revised version May 11, 1998; in final form August 3, 1998)

\begin{abstract}
An original method of treating the kinetic and exchange-correlation energies functionals in terms of many particle interactions was developed. It is based on utilizing the local density approximation. The total electron density, extracted from the $a b$ initio band structure calculations, is expressed as a linear superposition of contributions from the individual pseudoions embedded in the uniform background. The explicit expressions for the pair and triplet potentials are presented. The general form for the part of the pair interatomic interactions caused by the kinetic and the exchange-correlation effects is obtained. Relationship between the developing approach and the perturbation theory in pseudopotential is analysed. Unlike the perturbation theory the advanced approach allows one to calculate accurately the so-called reducible contributions to the pair potential arising from the $n$-particle $(n>2)$ interactions. It corresponds to summing certain series in pseudopotential. Contribution of the electron-nonlocal pseudopotential interactions to the pair interatomic ones is considered within the concept of the fully separable pseudopotentials.
\end{abstract}

PACS numbers: 71.15.Nc, 71.15.Hx, 34.20.Cf

\section{Introduction}

Calculation of interatomic potentials is one of the central problem in the modern condensed matter physics. It is caused by the utilizing of these potentials in many physics and materials science problems: construction of alloy statistical theory at the microscopic level $[1,2]$, computer simulations by the molecular dynamics method [3, 4], calculation of phonon spectra and properties related to them [5], etc. In some cases an adequate theoretical description of metal and alloy

*e-mail: zog@icmp.lviv.ua or gurski@math.uni.opole.pl

†e-mail: jekra@math.uni.opole.pl 
properties could not be achieved within the pair interatomic interaction model [6]. It means that the many-body interactions (three-, four-particles) play important role in these objects [7].

A consistent calculation of the many-body interatomic potentials is a complicated problem. One can distinguish two ways in solution of it.

1. The many particle interatomic potentials are being obtained within the pseudopotential theory $[1,3,8,9]$ in considering the high orders of the perturbation theory in pseudopotential (the third order and the fourth one) [6,7]. Serious difficulties arise in such a case related to an accurate account of all terms of the same order in pseudopotential.

2. Another way concerns derivation of the effective interatomic interactions in metallic alloys, see, for example [10-13].

Let us consider a binary system A-B. Ab initio studies of its properties are based on the Ising type Hamiltonian [14]

$$
\begin{aligned}
H= & N E_{0}+\sum_{\boldsymbol{R}} D_{\boldsymbol{R} \sigma_{\boldsymbol{R}}}+\frac{1}{2} \sum_{\boldsymbol{R}_{1}, \boldsymbol{R}_{2}} V_{2}\left(\boldsymbol{R}_{1}, \boldsymbol{R}_{2}\right) \sigma_{\boldsymbol{R}_{1}} \sigma_{\boldsymbol{R}_{2}} \\
& +\frac{1}{3} \sum_{\boldsymbol{R}_{1} \ldots \boldsymbol{R}_{3}} V_{\mathbf{3}}\left(\boldsymbol{R}_{1} \ldots \boldsymbol{R}_{3}\right) \sigma_{\boldsymbol{R}_{1}} \sigma_{\boldsymbol{R}_{2}} \sigma_{\boldsymbol{R}_{3}} \\
& +\frac{1}{4} \sum_{\boldsymbol{R}_{1} \ldots \boldsymbol{R}_{4}} V_{4}\left(\boldsymbol{R}_{1} \ldots \boldsymbol{R}_{4}\right) \sigma_{\boldsymbol{R}_{1}} \ldots \sigma_{\boldsymbol{R}_{4}}+\ldots
\end{aligned}
$$

Here $\sigma_{R}$ is an occupation number, which is equal to unity, if the site $\boldsymbol{R}$ is occupied by an atom of the kind $\mathrm{A}$, and $\sigma_{R}=0$ otherwise. The following notations are accepted in Eq. (1): $E_{0}$ is the energy per atom of the pure system $\mathrm{A}, N$ is the total number of atoms, $V_{2}, V_{3}, V_{4}$ denote the interactions of a pair, triplet, and quadruplet of atoms, respectively. The interatomic interactions in Eq. (1) are equal to zero by definition, if, at least, two site indices coincide. The parameters of the Hamiltonian (1), often called the effective cluster interactions, are usually determined by the Connolly-Williams method [14]. The crucial approximation is used in it that the parameters of (1) do not depend on alloy concentration and volume. Then, 5 parameters are searched from the total energy calculations performed for five ordered alloys, namely, two pure metals $A$ and $B$ and $A B, A_{3} B$ and $\mathrm{AB}_{3}$ compounds [14].

Each way has its advantages and restrictions. For example, approximation of the nearest neighbours is commonly used in the Connolly-Williams method. However, the nearest neighbour model cannot describe real alloy adequately, because the pair effective interatomic potentials in metals and alloys neither belong to the short-range nor to the long-range ones $[1,3]$. They need to take into account the interatomic interactions within, at least, 4 or 5 coordination spheres to reach reasonable results for metal and alloy properties $[1,3,6]$.

This brief review of literature shows clearly that determination of the many-body interatomic interactions in metals is an urgent problem.

A somewhat another approach to deriving the effective interatomic potentials in metals has been advanced in [15]. It is based on utilizing the Hohenberg-Kohn theorem [16] and treatment of the Kohn-Sham total energy functional [17] in 
terms of the many-body interatomic interactions. The further development of this approach is the aim of the present paper.

The paper is organized as follows. The main ideas of [15] are given in Sec. 2. Contribution of the electron-nonlocal pseudopotential interactions to the pair interatomic potential is analysed in Sec. 3. Calculations are performed for the case of fully separable pseudopotentials which possess the most general form in the class of the nonlocal pseudopotentials [1]. The explicit expression for the three-particle interatomic interactions is presented in Sec. 4. Conclusions, given in Sec. 5, complete the paper.

\section{The Kohn-Sham total energy functional and the many-body effective interatomic interactions}

Let us consider an electron-ion system within the pseudopotential concept. It means that pseudoions are placed in positions $\boldsymbol{R}$ and their interactions with the valence electrons are described by a pseudopotential $w(\boldsymbol{r}-\boldsymbol{R})$ which is an operator in à general case. form [17]:

The Kohn-Sham total energy functional of such a system has the following

$$
E[\rho]=T[\rho]+\int W(r) \rho(r) \mathrm{d} r+\frac{1}{2} \int \frac{\rho(r) \rho\left(r^{\prime}\right)}{\left|r-r^{\prime}\right|} \mathrm{d} r \mathrm{~d} r^{\prime}+E_{\mathbf{x c}}[\rho]+E_{\mathrm{i}-\mathrm{i}}
$$

(all equations are written in the atomic units in the present paper). Here $T[\rho]$ is a functional of the kinetic energy, the second and the third terms in (2) describe energy of the electron subsystem in the external field of the pseudoions

$$
W(r)=\sum_{\boldsymbol{R}} w(\boldsymbol{r}-\boldsymbol{R})
$$

and the Hartree energy, respectively. The last terms in (2) are $E_{\mathrm{xc}}$, the exchange-correlation energy functional and $E_{\mathrm{i}-\mathrm{i}}$, energy of the ion-ion direct interactions. The total energy $E[\rho](2)$ is the universal functional of an electron density

$$
\rho(r)=\sum_{\boldsymbol{k}} \Psi_{\boldsymbol{k}}^{*}(r) \Psi_{\boldsymbol{k}}(r)
$$

with $\Psi_{\boldsymbol{k}}(\boldsymbol{r})$ - the electron wave functions and $k$ - the wave vectors characterising electron states. Summation in (4) is performed over all occupied states.

The main problem in the Kohn-Sham formalism is finding the electron density $\rho(r)$. Some methods exist in the modern band structure theory [18] to find $\Psi_{\boldsymbol{k}}(r)$ solving the Kohn-Sham equations

$$
\left[-\frac{1}{2} \nabla^{2}+W(r)+V_{\mathrm{H}}(r)+V_{\mathrm{xc}}(r)\right] \Psi_{k}(r)=E_{k} \Psi_{k}(r),
$$

where $E_{k}$ is the Kohn-Sham eigenvalue,

$$
V_{\mathrm{H}}(\boldsymbol{r})=\int \frac{\rho\left(\boldsymbol{r}^{\prime}\right)}{\left|\boldsymbol{r}-\boldsymbol{r}^{\prime}\right|} d \boldsymbol{r}^{\prime}
$$

and

$$
V_{\mathrm{xc}}(\boldsymbol{r})=\frac{\delta E_{\mathrm{xc}}[\rho(r)]}{\delta \rho(\boldsymbol{r})}
$$


are the Hartree $V_{\mathbf{H}}$ and the exchange-correlation $V_{\mathbf{x c}}$ potentials. Besides, several efficient techniques have been recently developed for calculation of the $\rho(r)$ without direct finding of the $\Psi_{\boldsymbol{k}}(\boldsymbol{r})$, see review [19]. Thus, the present theory provides knowledge on the electron density $\rho(r)$, that minimizes $E[\rho(r)](2)$, with the high accuracy $[18,19]$.

Let us suppose the eigenvalue problem (5) is solved self-consistently by one of the conventional band structure theory methods [18], that is the total valence electron density $\rho(\boldsymbol{r})$ is determined. In Eq. [15] the known quantity of $\rho(r)$ has been presented in the form

$$
\rho(r)=\rho_{0}+\sum_{\boldsymbol{R}} \rho_{i}(\boldsymbol{r}-\boldsymbol{R})
$$

where $\rho_{0}=z / \Omega_{0}\left(\Omega_{0}=\Omega / N\right)$ is a density of the uniform electron distribution, $z$ an ion valency, $\Omega_{0}$ the atomic volume and $\rho_{i}(r-R)$ is an electron density related to the pseudoion at the site $\boldsymbol{R}$. Equation (8) indicates that the total electron density in a metal is represented as the linear superposition of contributions from the individual pseudoions, embedded in the uniform background of the density $\rho_{0}$.

We assume that $\rho_{i}(\boldsymbol{r}-\boldsymbol{R})$ in (8) do not depend on metal structure, that is, absolute values of the lattice sites $\boldsymbol{R}$. The explicit analytical expressions for $\rho_{i}(\boldsymbol{r}-\boldsymbol{R})$ obtained within the perturbation theory in pseudopotential substantiate this assumption: $\rho_{i}(\boldsymbol{r}-\boldsymbol{R}) \equiv \rho_{i}(|\boldsymbol{r}-\boldsymbol{R}|)[1,7,9]$. The structure dependence of the total electron density $\rho(r)$ is achieved in (8) through summation over ion positions.

The focal point of the linear superposition assumption (8) is an accurate real-space treatment of the metal total energy in terms of well-defined interatomic potentials [15]. Really, Eq. (8) allows one to develop the total energy of a metal as a multi-ion expansion involving its $N$ individual ion coordinates $\boldsymbol{R}[7,15]$, that is

$$
E_{\mathrm{tot}}=E_{0}\left(\Omega_{0}\right)+\frac{1}{2 !} \sum_{i, j}^{\prime} V_{2}\left(\boldsymbol{R}_{i}, \boldsymbol{R}_{j}\right)+\frac{1}{3 !} \sum_{i, j, l}^{\prime} V_{3}\left(\boldsymbol{R}_{i}, \boldsymbol{R}_{j}, \boldsymbol{R}_{l}\right)+\ldots
$$

where $E_{0}$ represents a volume term including one-ion contributions and $V_{2}, V_{3}$ etc. are the two-particle, three-particle etc. interatomic potentials, which are implicitly volume dependent, but explicitly structure independent $[7,15]$. To put it in another way the potentials themselves are independent of the absolute ion positions $\boldsymbol{R}_{i}$ and depend only on relative separations $R_{i j}=\left|\boldsymbol{R}_{i}-\boldsymbol{R}_{j}\right|$ [7], for example, the angular-force triplet potential $V_{3}$ is the three-dimensional function: $V_{3}\left(\boldsymbol{R}_{1}, \boldsymbol{R}_{2}, \boldsymbol{R}_{3} ; \Omega\right) \equiv V_{3}\left(R_{12}, R_{13}, R_{23} ; \Omega\right)$ [7]. The prime on the each summation in (9) denotes exclusion of all the self-interaction terms where two indices are equal. The interatomic potentials $V_{2}, V_{3}$ etc. present on the right hand side of Eq. (9) are completely transferable at fixed volume because of their independence on metal structure $[3,7-9]$. The structure dependence of the total energy (9) appears through the summations in (9) over all $N$ ion positions.

One should stress once more this important statement. The total electron density $\rho(r)$ is determined for the periodic system in the both approaches mentioned above. In Refs. [7-9] it is searched analytically as expansion (8) within the perturbation theory in pseudopotential. We propose to evaluate the metal total energy and electron density performing $a b$ initio band structure calculations and then, represent $E_{\text {tot }}$ in the form of Eq. (9) using assumption (8). The essential 
feature of such a procedure is the following one. The interatomic potentials entering expansion (9) are volume dependent only and thus rigorously transferable at a given volume to all bulk structures, either ordered or disordered [7].

One should treat the $T[\rho]$ and $E_{\mathrm{xc}}[\rho]$ functionals in terms of contributions to the many-body interatomic interactions to get the totál energy in the form (9).

The local density approximation (LDA) [17] is almost universally used for the $E_{\text {xc }}[\rho]$ functional in total energy pseudopotential calculations $[18,19]$. According to the LDA

$$
E_{\mathrm{xc}}[\rho]=\int \mathrm{d} \boldsymbol{r} \rho(r) \varepsilon_{\mathrm{xc}}(\rho(r))
$$

with $\varepsilon_{\mathrm{xc}}(\rho(r))$, the exchange-correlation energy per electron at point $r$ which is equal to the exchange-correlation energy of the homogeneous electron gas possessing the same density $\rho(r)$ as the electron subsystem under investigation. On explicit expressions for $\varepsilon_{\mathrm{xc}}(\rho(\boldsymbol{r}))$ see [20,21]. Expression (10) can be transformed in the following manner [15]:

$$
\begin{array}{r}
E_{\mathrm{xc}}[\rho]=E_{\mathrm{xc}}^{(0)}+\frac{1}{2 !} \sum_{\boldsymbol{R}_{1}, \boldsymbol{R}_{2}}^{\prime} V_{\mathrm{xc}}^{(2)}\left(\boldsymbol{R}_{1}, \boldsymbol{R}_{2}\right) \\
+\frac{1}{3 !} \sum_{\boldsymbol{R}_{1}-\boldsymbol{R}_{3}}^{\prime} V_{\mathrm{xc}}^{(3)}\left(\boldsymbol{R}_{1}, \boldsymbol{R}_{2}, \boldsymbol{R}_{3}\right)+\ldots
\end{array}
$$

where $E_{\mathrm{xc}}^{(0)}$ is the structure independent part of the exchange-correlation energy and $V_{\mathrm{xc}}^{(2)}\left(\boldsymbol{R}_{1}, \boldsymbol{R}_{2}\right), V_{\mathrm{xc}}^{(3)}\left(\boldsymbol{R}_{1} \ldots \boldsymbol{R}_{3}\right)$ etc. are appropriate contributions to the pair, triplet etc. indirect interatomic interactions caused by the exchange-correlation effects. On explicit formulae for $E_{\mathrm{xc}}^{(0)}, V_{\mathrm{xc}}^{(2)}, V_{\mathrm{xc}}^{(3)}$, see [15].

Consider the kinetic energy functional $T[\rho]$. Within the pseudopotential concept the unknown $\Psi_{k}(r)$ functions of the valence electrons are searched on the plane-wave basis $[1,3,19]$, that is

$$
\Psi_{\boldsymbol{k}}(r)=\sum_{\boldsymbol{G}} a_{\boldsymbol{G}}(k) \exp [\mathrm{i}(k+\boldsymbol{G}) \boldsymbol{r}]
$$

Here $a_{G}(k)$ are the expansion coefficients and $\boldsymbol{G}$ the reciprocal lattice vectors. Solution of the Kohn-Sham equations (5) provides information on coefficients $a_{G}(k)$ as eigenvectors of this problem. The valence electron kinetic energy with allowance for (12) is equal to

$$
T=\sum_{\boldsymbol{k}} \sum_{\boldsymbol{G} \leq \boldsymbol{G}_{\mathrm{M}}} \frac{1}{2}\left|a_{G}(\boldsymbol{k})\right|^{2} \cdot|k+\boldsymbol{G}|^{2} .
$$

Summation in $k$ is performed over the occupied states. The $\boldsymbol{G}_{\mathbf{M}}$ vector is determined by the cutoff energy $\left|k+G_{M}\right|^{2} / 2$. Let us represent the known quantity $T(13)$ as follows:

$$
\sum_{\boldsymbol{k}} \sum_{\boldsymbol{G}} \frac{1}{2}\left|a_{\boldsymbol{G}}(\boldsymbol{k})\right|^{2} \cdot|\boldsymbol{G}+k|^{2}=\int \mathrm{d} \boldsymbol{r} \rho(\boldsymbol{r}) t(\rho(\boldsymbol{r})) .
$$

that is in the form of LDA. Then the kinetic energy could be rewritten like $E_{\mathrm{xc}}[\rho](11)$. One can regard (14) as a constraint on the unknown function $t(\rho(r))$. 
In the case of simple (nontransition) metals the following form could be used for the $t(\rho(r))$ function:

$$
t(\rho(r))=C \frac{3}{10}\left[3 \pi^{2} \rho(r)\right]^{2 / 3} .
$$

The value $C=1$ corresponds to the homogeneous electron gas LDA expression. We propose to treat $C$ as a free coefficient fitting the right hand side of Eq. (14) to the valence electron kinetic energy value (13).

The pseudopotential concept permits to exclude the core electrons from the explicit consideration but the true electron-ion potential is replaced by a nonlocal operator that describes an effective electron-ion interaction $[1,3]$. On application of the approximation (14) to the transition metals and possible forms for $t(r ;[\rho])$ functional see. [22] and literature cited there.

One should emphasiz: once more that the approximation (14) with (15) is used just for interpretation of the valence electron kinetic energy in terms of appropriate contributions to the many-ion indirect interactions in (9). The electron density (4) is calculated by the conventional band-structure methods $[18,19]$ from the solution of the Kohn-Sham equations (5) using there the LDA expression for $V_{\mathrm{xc}}(\boldsymbol{r})$.

We expound briefly the main ideas of the approach advanced in [15].

The regular metal of the valency $z$ with one atom per primitive cell is considered. The functions $t(r)$ and $\varepsilon_{\mathrm{xc}}(r)$ are periodical ones in the regular crystal because of the electron density $\rho(r)$ periodicity. Therefore, one should consider them in the region of the Wigner-Seitz cell only. Let us define

$$
\rho_{\mathrm{ps}}\left(\boldsymbol{r}-\boldsymbol{R}_{1}\right)=\rho_{0}+\rho_{\mathrm{i}}\left(\boldsymbol{r}-\boldsymbol{R}_{1}\right)
$$

as the electron density of a pseudoatom at the site $\boldsymbol{R}_{1}$ and expand $t(\rho(\boldsymbol{r}))$ and $\varepsilon_{\mathrm{xc}}(\rho(\boldsymbol{r}))$ in $\rho_{\mathrm{ps}}\left(\boldsymbol{r}-\boldsymbol{R}_{1}\right)$ for $\boldsymbol{r} \in \Omega_{0}(1)$. Symbol $\boldsymbol{r} \in \Omega_{0}(1)$ denotes that the running variable $r$ takes values in the unit cell (the Wigner-Seitz cell) centred at the site $\boldsymbol{R}_{1}$. For function $f(\rho(\boldsymbol{r}))=t(\rho(r))$ and $f(\rho(\boldsymbol{r}))=\varepsilon(\rho(\boldsymbol{r}))$ such an expansion reads

$$
\begin{gathered}
f(\rho(r))=f\left(\rho_{\mathrm{ps}}\left(\boldsymbol{r}-\boldsymbol{R}_{1}\right)\right)+\frac{\partial f\left(r-\boldsymbol{R}_{1}\right)}{\partial \rho} \sum_{\boldsymbol{R} \neq \boldsymbol{R}_{1}} \rho_{i}(r-\boldsymbol{R}) \\
+\frac{1}{2 !} \frac{\partial^{2} f\left(\boldsymbol{r}-\boldsymbol{R}_{1}\right)}{\partial \rho^{2}}\left[\sum_{\boldsymbol{R} \neq \boldsymbol{R}_{1}} \rho_{i}(r-\boldsymbol{R})\right]^{2}+\ldots
\end{gathered}
$$

with $r \in \Omega_{0}(1)$ and

$$
\left.\frac{\partial^{n} f\left(\boldsymbol{r}-\boldsymbol{R}_{1}\right)}{\partial \rho^{n}} \equiv \frac{\partial^{n} f(\rho(\boldsymbol{r}))}{\partial \rho^{n}}\right|_{\rho(r)=\rho_{\mathrm{ps}}\left(\boldsymbol{r}-\boldsymbol{R}_{1}\right)} .
$$

It is seen from (8) and (16) that the total electron density $\rho(r)$ in the vicinity of each atom is expressed as a sum of the density $\rho_{\mathrm{ps}}$ contributed by the pseudoatom in a question plus contributions from the surrounding ions that is

$$
\rho(r)=\rho_{\mathrm{ps}}\left(\boldsymbol{r}-\boldsymbol{R}_{j}\right)+\sum_{\boldsymbol{R} \neq \boldsymbol{R}_{j}} \rho_{i}(r-\boldsymbol{R}) \quad \text { for } \quad r \in \Omega_{0}(j) .
$$


The condition

$$
\left|\sum_{\boldsymbol{R} \neq \boldsymbol{R}_{j}} \rho_{i}(r-\boldsymbol{R}) / \rho_{\mathrm{ps}}\left(r-\boldsymbol{R}_{\mathrm{j}}\right)\right| \ll 1 \quad \text { for } \quad r \in \Omega_{0}(j)
$$

provides convergence of the series for the $\rho(r) t(\rho(r))$ and the $\rho(r) \varepsilon(\rho(r))$. Thus, it is the main trick to represent the known total electron density $\rho(r)$ in the each unit cell $\Omega_{0}(j)$ in such a manner (see Eq. (19)) that the condition (20) would take place in the whole $\Omega_{0}(j)$ region.

The kinetic and the exchange-correlation energies are functionals of the total electron density. Owing to the (10) and (14) one can treat the $E_{\mathrm{xc}}[\rho]$ and the $T[\rho]$ in terms of contributions of the exchange-correlation and the kinetic energy effects to the many-body interatomic indirect interactions [15]. For example, the kinetic energy takes the following form with allowance for (14) and (17) to (19):

$$
\begin{aligned}
& \int_{\Omega} \rho(r) t(\rho(r)) \mathrm{d} r=N\left[\int_{\Omega_{0}(1)} \mathrm{d} r \rho_{\mathrm{ps}}\left(r-\boldsymbol{R}_{1}\right) t\left(r-\boldsymbol{R}_{1}\right)\right. \\
& \left.\quad+\frac{1}{2 !} \sum_{\boldsymbol{R}_{2}}^{\prime} V_{\mathrm{kin}}^{(2)}\left(\boldsymbol{R}_{1}, \boldsymbol{R}_{2}\right)+\frac{1}{3 !} \sum_{\boldsymbol{R}_{2}, \boldsymbol{R}_{3}}^{\prime} V_{\mathrm{kin}}^{(3)}\left(\boldsymbol{R}_{1}, \boldsymbol{R}_{2}, \boldsymbol{R}_{3}\right)+\ldots\right]
\end{aligned}
$$

Here symbol $\int_{\Omega_{0}(1)}$ denotes integration over the unit cell. The first term on the right hand side of Eq. (21) is this part of the kinetic energy which contributes to the $E_{0}\left(\Omega_{0}\right)$, volume dependent part of the total energy, see (9). One can interpret it as the kinetic energy of the interacting electron gas. The second and the third (etc.) terms on the right hand side of (21) form the band structure energy. The appropriate contributions of the $V_{\text {kin }}^{(2)}\left(\boldsymbol{R}_{1}, \boldsymbol{R}_{2}\right), V_{\mathrm{kin}}^{(3)}\left(\boldsymbol{R}_{1}, \boldsymbol{R}_{2}, \boldsymbol{R}_{3}\right)$ etc. to the pair, triplet etc. interatomic indirect interactions could be obtained easily by utilizing Eqs. (14) and (17) to (19).

The exchange-correlation energy functional is treated in the same manner [15].

Any known pseudopotential can be written as a sum of local and nonlocal parts $[1,3,19]$

$$
w(r-\boldsymbol{R})=w^{\mathrm{loc}}(\boldsymbol{r}-\boldsymbol{R})+w^{\mathrm{nl}}(\boldsymbol{r}-\boldsymbol{R}) .
$$

Treatment of the electrostatic energy in terms of interatomic potentials is described in detail in [3, 7-9]. The corresponding components of the second, third, and the last terms in (2) related to the uniform electron distribution $\rho_{0}$ and the point charges of ions form the Ewald energy, see [7, 15]. It pays to note that the electrostatic interactions contribute only to the structure independent term $E_{0}\left(\Omega_{0}\right)$ and the pair potentials $V_{2}$ in (9). Calculation of these contributions is not an original point of the present paper. That is why omitting details of derivation given in [15] (see also [7-9]) and using (22) we present the final expression for the pair interatomic potential in metal 


$$
\begin{aligned}
& V_{2}\left(\boldsymbol{R}_{1}, \boldsymbol{R}_{2}\right)=V_{\mathrm{i}-\mathrm{i}}^{\mathrm{dir}}\left(\boldsymbol{R}_{1}, \boldsymbol{R}_{2}\right)+\frac{2 \Omega_{0}^{2}}{(2 \pi)^{3}} \int \mathrm{d}^{3} q\left[w^{\mathrm{loc}}(q)+\frac{1}{2} V_{\mathrm{H}}(q)\right] \rho_{i}(-q) \\
& \times \exp \left[\mathrm{i} \boldsymbol{q}\left(\boldsymbol{R}_{1}-\boldsymbol{R}_{2}\right)\right]+V_{2}^{\mathrm{nl}}\left(\boldsymbol{R}_{1}, \boldsymbol{R}_{2}\right)+\left[V_{\mathrm{xc}}^{(2)}\left(\boldsymbol{R}_{1}, \boldsymbol{R}_{2}\right)+V_{\mathrm{kin}}^{(2)}\left(\boldsymbol{R}_{1}, \boldsymbol{R}_{2}\right)\right] .
\end{aligned}
$$

The following notations are used in $(23): V_{i-i}^{\text {dir }}\left(\left|\boldsymbol{R}_{1}-\boldsymbol{R}_{2}\right|\right)$ is a potential of the direct ion-ion interaction,

$$
V_{\mathrm{H}}(q)=\frac{4 \pi \rho_{\mathrm{ps}}(q)}{q^{2}}
$$

$\rho_{\mathrm{ps}}(q)$ and $w^{\mathrm{loc}}(q)$ the Fourier transforms of the $\rho_{\mathrm{ps}}(\boldsymbol{r}-\boldsymbol{R})$ and $w^{\mathrm{loc}}(\boldsymbol{r}-\boldsymbol{R})$, respectively. A contribution of the electron-nonlocal pseudopotential interactions to the pair interatomic potential, $V_{2}^{\text {nl }}\left(\boldsymbol{R}_{1}, \boldsymbol{R}_{2}\right)$, is considered in detail in the next section.

Using a conventional in the pseudopotential method procedure we have obtained the structure independent and transferable pair potentials (23) as a function of an ion separation $[1,3,7]$. Application of these potentials in the metal phonon spectrum calculations or in computer simulations within the molecular dynamics method may provide a convincing verification of the approach proposed.

The general formulae for the $V_{\mathrm{xc}}^{(2)}\left(\boldsymbol{R}_{1}, \boldsymbol{R}_{2}\right)$ and $V_{\text {kin }}^{(2)}\left(\boldsymbol{R}_{1}, \boldsymbol{R}_{2}\right)$ terms (see (23)) are derived in the present paper.

$$
\begin{aligned}
& \frac{1}{2 !}\left[V_{\mathrm{xc}}^{(2)}\left(\boldsymbol{R}_{1}, \boldsymbol{R}_{2}\right)+V_{\mathrm{kin}}^{(2)}\left(\boldsymbol{R}_{1}, \boldsymbol{R}_{2}\right)\right] \\
& =\int_{\Omega_{0}(1)}\left[V_{\mathrm{xc}}\left(\boldsymbol{r}-\boldsymbol{R}_{1}\right)+V_{\mathrm{kin}}\left(r-\boldsymbol{R}_{1}\right)\right] \rho_{i}\left(\boldsymbol{r}-\boldsymbol{R}_{2}\right) \mathrm{d} \boldsymbol{r} \\
& +\frac{1}{2 !} \int_{\Omega_{0}(1)}\left[\frac{\partial V_{\mathrm{xc}}\left(\boldsymbol{r}-\boldsymbol{R}_{1}\right)}{\partial \rho}+\frac{\partial V_{\mathrm{kin}}\left(r-\boldsymbol{R}_{1}\right)}{\partial \rho}\right] \rho_{i}^{2}\left(\boldsymbol{r}-\boldsymbol{R}_{2}\right) \mathrm{d} \boldsymbol{r}+\ldots \\
& +\frac{1}{n !} \int_{\Omega_{0}(1)}\left[\frac{\partial^{(n-1)} V_{\mathrm{xc}}\left(\boldsymbol{r}-\boldsymbol{R}_{1}\right)}{\partial \rho^{(n-1)}}+\frac{\partial^{(n-1)} V_{\mathrm{kin}}\left(\boldsymbol{r}-\boldsymbol{R}_{1}\right)}{\partial \rho^{(n-1)}}\right] \rho_{i}^{n}\left(\boldsymbol{r}-\boldsymbol{R}_{2}\right) \mathrm{d} \boldsymbol{r}+\ldots
\end{aligned}
$$

where, see (7), (10) and (14)

$$
V_{\mathrm{xc}}\left(\boldsymbol{r}-\boldsymbol{R}_{1}\right)=\left.\frac{\partial}{\partial \rho}\left[\rho(\boldsymbol{r}) \varepsilon_{\boldsymbol{x} c}(\rho(\boldsymbol{r}))\right]\right|_{\rho(\boldsymbol{r})=\rho_{\mathrm{ps}}\left(\boldsymbol{r}-\boldsymbol{R}_{1}\right)}
$$

and

$$
V_{\text {kin }}\left(r-\boldsymbol{R}_{1}\right)=\left.\frac{\partial}{\partial \rho}[\rho(r) t(\rho(\boldsymbol{r}))]\right|_{\rho(\boldsymbol{r})=\rho_{\mathrm{ps}}\left(\boldsymbol{r}-\boldsymbol{R}_{1}\right)} .
$$

The terms of a kind $\partial^{(n)}\left[V_{\mathrm{xc}}+V_{\mathrm{kin}}\right] / \partial \rho^{(n)}, n=1,2 \ldots$ on the right hand side of (25) correspond to the contributions to the pair indirect interatomic interactions arising from the reducible parts of the perturbation theory series in pseudopotential. They should emphasize that the present stage of the metal microscopic theory permits one to estimate the reducible terms of the third and the fourth order in pseudopotential only $[3,6]$. Unlike the perturbation theory in pseudopotential, the developing approach allows one to work out in a general form a procedure for 
renormalization of the $n$-particle irreducible interactions by the $(n+1)-,(n+2)$ etc. reducible ones. Such a renormalization of the pair interatomic potential is given in this section, see (23) and (25). One can prove easily using the explicit expressions for $t(\rho(r)), \varepsilon_{\mathrm{xc}}[\rho]$ and Eq. (25) that the series in (25) is a convergent one, if the inequality (20) takes place in the unit cell region. To put it in another way, in the unit cell centred, for example, at the site $\boldsymbol{R}_{1}$, the electron density distributions $\rho_{i}(\boldsymbol{r}-\boldsymbol{R}),\left(\boldsymbol{R} \neq \boldsymbol{R}_{1}\right)$, related to the nearest neighbours of an atom in the site $\boldsymbol{R}_{1}$, must be small in comparison with $\rho_{\mathrm{ps}}\left(r-\boldsymbol{R}_{1}\right)(16)$.

The case of the triplet indirect interactions is considered in Sec. 4.

\section{The electron-nonlocal pseudopotential interactions}

One should analyse the term $V_{2}^{\mathrm{nl}}\left(\boldsymbol{R}_{1}, \boldsymbol{R}_{2}\right)$ of Eq. (23) to define the pair interatomic potential completely. The case of the fully separable pseudopotentials [23] possessing the most general form in the class of nonlocal potentials is considered in the present paper

$$
\begin{aligned}
& W^{\mathrm{nl}}\left(r, r^{\prime}\right)=\sum_{\boldsymbol{R}} w^{\mathrm{nl}}\left(r-\boldsymbol{R}, \boldsymbol{r}^{\prime}-\boldsymbol{R}\right), \\
& w^{\mathrm{nl}}\left(r-\boldsymbol{R}, \boldsymbol{r}^{\prime}-\boldsymbol{R}\right)=\sum_{l, m} Q_{l} \zeta_{l}(|r-\boldsymbol{R}|) \zeta_{l}\left(\left|r^{\prime}-\boldsymbol{R}\right|\right) \\
& \quad \times Y_{l m}^{*}\left(\theta_{r-R}, \varphi_{r-\boldsymbol{R}}\right) Y_{l m}\left(\theta_{r^{\prime}-R}, \varphi_{r^{\prime}-R}\right) .
\end{aligned}
$$

Here $Q_{l}$ are parameters of the $w^{\mathrm{nl}}$, the short-range functions $\zeta_{l}(|r-\boldsymbol{R}|)$ depend on $r-\boldsymbol{R}$ vector modulus and are different for different values of $l, Y_{l m}(\theta, \varphi)$ are the spherical harmonics with the quantum numbers $l, m$ characterizing the angular momentum and $\theta_{\boldsymbol{r}-\boldsymbol{R}}, \varphi_{\boldsymbol{r}-\boldsymbol{R}}$ and $\theta_{\boldsymbol{r}^{\prime}-\boldsymbol{R}}, \varphi_{\boldsymbol{r}^{\prime}-\boldsymbol{R}}$, the spherical angles of $\boldsymbol{r}-\boldsymbol{R}$ and $\boldsymbol{r}^{\prime}-\boldsymbol{R}$ vectors, respectively.

Contribution of the electron-nonlocal pseudopotential interactions to the total energy is the following one:

$$
E^{\mathrm{nl}}=\int_{\Omega} W^{\mathrm{nl}}\left(r, r^{\prime}\right) \rho\left(r, r^{\prime}\right) \mathrm{d} r \mathrm{~d} \boldsymbol{r}^{\prime}
$$

where, compare with (4) and (8)

$$
\rho\left(r, r^{\prime}\right)=\sum_{\boldsymbol{k}} \Psi_{\boldsymbol{k}}^{*}(r) \Psi_{\boldsymbol{k}}\left(r^{\prime}\right)=\sum_{k} \sum_{\boldsymbol{R}} \psi_{i, \boldsymbol{k}}^{*}(\boldsymbol{r}-\boldsymbol{R}) \psi_{i, k}\left(\boldsymbol{r}^{\prime}-\boldsymbol{R}\right)+\frac{z}{\Omega_{0}}
$$

Then

$$
\rho_{\mathrm{ps}}\left(r-\boldsymbol{R}, \boldsymbol{r}^{\prime}-\boldsymbol{R}\right)=\frac{z}{\Omega_{0}}+\sum_{\boldsymbol{k}} \psi_{i, k}^{*}(r-\boldsymbol{R}) \psi_{i, k}\left(\boldsymbol{r}^{\prime}-\boldsymbol{R}\right)
$$

is the element of the electron density matrix related to the pseudoion at the site $\boldsymbol{R}$.

Substitute (31) and (32) to (30). Then

$$
E^{\mathrm{nl}}=E_{0}^{\mathrm{nl}}\left(\Omega_{0}\right)+\frac{1}{2} \sum_{\boldsymbol{R}_{1}, \boldsymbol{R}_{2}} V_{2}^{\mathrm{nl}}\left(\boldsymbol{R}_{1}, \boldsymbol{R}_{2}\right)
$$

The volume dependent part of the $E^{\mathrm{nl}}$, which contributes to the $E_{0}\left(\Omega_{0}\right)$, see $(9)$, equals

$$
E_{0}^{\mathrm{n} l}\left(\Omega_{0}\right)=\sum_{\boldsymbol{R}} \int_{\Omega_{0}(\boldsymbol{R})} \mathrm{d} r \mathrm{~d} \boldsymbol{r}^{\prime} w^{\mathrm{nl}}\left(\boldsymbol{r}-\boldsymbol{R}, \boldsymbol{r}^{\prime}-\boldsymbol{R}\right) \rho_{\mathrm{ps}}\left(\boldsymbol{r}-\boldsymbol{R}, \boldsymbol{r}^{\prime}-\boldsymbol{R}\right) .
$$


The procedure of $V_{2}^{\mathrm{nl}}\left(\boldsymbol{R}_{1}, \boldsymbol{R}_{2}\right)$ calculation is given in [15]. It may be generalized easily to the case of the fully scprarable pseudopotentials. The final result has the form

$$
\begin{gathered}
\frac{1}{2} V_{2}^{\mathrm{n} l}\left(\boldsymbol{R}_{1}, \boldsymbol{R}_{2}\right)=\sum_{l m} Q_{l} \frac{1}{N} \sum_{\boldsymbol{k}, n \leq k_{\mathrm{F}}}\left[\sum_{m^{\prime}} \sum_{\boldsymbol{G}} 4 \pi \mathrm{i}^{\mathrm{l}} D_{m^{\prime} m}^{l}(0, \beta, 0) \psi_{i, k n}(\boldsymbol{G})\right. \\
\left.\times Y_{l m^{\prime}}\left(\theta_{\boldsymbol{G}}, \varphi_{\boldsymbol{G}}\right) \int_{0}^{\infty} \zeta_{l}\left(\left|\boldsymbol{r}-\boldsymbol{R}_{1}\right|\right) J_{l}\left(|\boldsymbol{G}| \cdot\left|\boldsymbol{r}-\boldsymbol{R}_{2}\right|\right) r^{2} \mathrm{~d} \boldsymbol{r}\right]^{2}
\end{gathered}
$$

The following notations are used in Eq. (35). $D_{m^{\prime} m}^{l}(\alpha, \beta, \gamma)$ are the generalized spherical harmonics (the Wigner function) depending on the three Eulerian angles, $\beta$ is an angle between $\boldsymbol{R}_{1}$ and $\boldsymbol{R}_{2}$ vectors, $\psi_{i, \boldsymbol{k} n}(\boldsymbol{G})$ is the Fourier transform of $\psi_{i, \boldsymbol{k} n}(\boldsymbol{r}-\boldsymbol{R}), \theta_{\boldsymbol{G}}, \varphi_{\boldsymbol{G}}$ are spherical angles of the reciprocal lattice vector $\boldsymbol{G}$ and $J_{l}(q r)$ is the Bessel function. Summation in (35) is performed over all the occupied states $k, n \leq k_{\mathrm{F}}$, where $k_{\mathrm{F}}$ is the Fermi momentum and $n$ index of the occupied electron zones. The sums with respect to $l$ and $\boldsymbol{G}$ are the rapid convergent ones owing to the dependences of the Bessel function $J_{l}(q r)$ on $l$ and the overlapping integral

$$
\int_{0}^{\infty} \zeta_{l}\left(\left|\boldsymbol{r}-\boldsymbol{R}_{1}\right|\right) J_{l}\left(|\boldsymbol{G}| \cdot\left|\boldsymbol{r}-\boldsymbol{R}_{2}\right|\right) r^{2} \mathrm{~d} \boldsymbol{r}
$$

on $\boldsymbol{G}$, respectively. One can show analysing Eq. (36) that the potential $V_{2}^{\text {nl }}\left(\boldsymbol{R}_{1}, \boldsymbol{R}_{2}\right)$ is the short-range one. That is why the contribution of $V_{2}^{\mathrm{nl}}\left(\boldsymbol{R}_{1}, \boldsymbol{R}_{2}\right)$ to the total energy is small $[1,3]$. However, this part of $E_{\text {tot }}(9)$ displays itself in more pronounced way at investigation of the equation of state, phonon spectra, and other properties which are derivatives of $E_{\text {tot }}$ with respect to $\Omega_{0}[1,3]$.

\section{Three-atom interactions in metal}

We restrict ourselves to investigating the three-particle interactions in this section. Deriving other many-body potentials does not face any difficulties. One can get with allowance for (10), (14) and (17) to (21) that

$$
\begin{aligned}
& \frac{1}{3 !} V_{3}\left(\boldsymbol{R}_{1}, \boldsymbol{R}_{2}, \boldsymbol{R}_{3}\right) \\
& =\frac{1}{2 !} \int_{\Omega_{\mathrm{o}}(1)}\left[\frac{\partial V_{\mathrm{kin}}\left(\boldsymbol{r}-\boldsymbol{R}_{1}\right)}{\partial \rho}+\frac{\partial V_{\mathrm{xc}}\left(r-\boldsymbol{R}_{1}\right)}{\partial \rho}\right] \rho_{i}\left(r-\boldsymbol{R}_{2}\right) \rho_{i}\left(r-\boldsymbol{R}_{3}\right) \mathrm{d} \boldsymbol{r} \\
& +\frac{C_{3}^{1}}{3 !} \int_{\Omega_{\mathrm{o}(1)}}\left[\frac{\partial^{2} V_{\mathrm{kin}}\left(\boldsymbol{r}-\boldsymbol{R}_{1}\right)}{\partial \rho^{2}}+\frac{\partial^{2} V_{\mathrm{xc}}\left(\boldsymbol{r}-\boldsymbol{R}_{1}\right)}{\partial \rho^{2}}\right] \rho_{i}^{2}\left(r-\boldsymbol{R}_{2}\right) \rho_{i}\left(\boldsymbol{r}-\boldsymbol{R}_{3}\right) \mathrm{d} \boldsymbol{r} \\
& +\frac{C_{4}^{1}}{4 !} \int_{\Omega_{\mathrm{o}(1)}}\left[\frac{\partial^{3} V_{\mathrm{kin}}\left(\boldsymbol{r}-\boldsymbol{R}_{1}\right)}{\partial \rho^{3}}+\frac{\partial^{3} V_{\mathrm{xc}}\left(\boldsymbol{r}-\boldsymbol{R}_{1}\right)}{\partial \rho^{3}}\right] \rho_{i}^{3}\left(r-\boldsymbol{R}_{2}\right) \rho_{i}\left(\boldsymbol{r}-\boldsymbol{R}_{3}\right) \mathrm{d} \boldsymbol{r} \\
& +\frac{C_{4}^{2}}{4 !} \int_{\Omega_{0}(1)}\left[\frac{\partial^{3} V_{\mathrm{kin}}\left(\boldsymbol{r}-\boldsymbol{R}_{1}\right)}{\partial \rho^{3}}+\frac{\partial^{3} V_{\mathrm{xc}}\left(\boldsymbol{r}-\boldsymbol{R}_{1}\right)}{\partial \rho^{3}}\right] \rho_{i}^{2}\left(\boldsymbol{r}-\boldsymbol{R}_{2}\right) \rho_{i}^{2}\left(\boldsymbol{r}-\boldsymbol{R}_{3}\right) \mathrm{d} \boldsymbol{r} \\
& +\ldots
\end{aligned}
$$


where $V_{\mathrm{kin}}\left(\boldsymbol{r}-\boldsymbol{R}_{1}\right)$ and $V_{\mathrm{xc}}\left(\boldsymbol{r}-\boldsymbol{R}_{1}\right)$ are defined by (27) and (26), respectively, $C_{n}^{i}$ is the binomial coefficient. Integration in (37) is performed over the Wigner-Seitz cell centred at the site $\boldsymbol{R}_{1}$. The first term on the right hand side of Eq. (37) represents the irreducible three-body potential while the next ones arise from the $n$-particle $(n>3)$ reducible interactions in a metal. For example, the third and the fourth terms in (37) have their origin in five-ion interactions, when some indices of ion coordinates coincide.

Equation (37) is written to an accuracy of $\rho_{i}^{4}(r-\boldsymbol{R})$, where $\rho_{i}(r-\boldsymbol{R})$ are the electron density fluctuations with respect to $\rho_{0}$ related to the neighbours of an ion at site $\boldsymbol{R}_{1}\left(\boldsymbol{R} \neq \boldsymbol{R}_{1}\right)$. One should consider $\rho_{i}(\boldsymbol{r}-\boldsymbol{R})$ as a small parameter in comparison with $\rho_{\mathrm{ps}}\left(\boldsymbol{r}-\boldsymbol{R}_{1}\right)$ with accordance to (20). It is seen from (37) that the three-particle interactions in a metal are indirect ones and caused by the band structure (the kinetic energy) and the exchange-correlation effects.

\section{Conclusions}

A scheme to deriving the effective interatomic potentials has been developed. The knowledge of the total electron density $\rho(r)$ is demanded only. The $\rho(r)$ can be obtained by different methods within the $a b$ initio band structure calculations. In the unit lattice cell the $\rho(\boldsymbol{r})$ is represented as a sum of the density $\rho_{\mathrm{ps}}\left(\boldsymbol{r}-\boldsymbol{R}_{j}\right)$ related to the atom at the centre of this cell plus contributions $\rho_{i}(\boldsymbol{r}-\boldsymbol{R})$ $\left(\boldsymbol{R} \neq \boldsymbol{R}_{j}\right)$ from the surrounding atoms, see (19). The $\rho_{i}(\boldsymbol{r}-\boldsymbol{R})$ are supposed to be independent on metal structure (absolute values of the ion positions $\boldsymbol{R}$ ).

The $\rho(r)$ expansion in contributions of the individual atoms is the essential point of the presenting method. The linear superposition assumption (19) for the total electron density permits one to get the analytical expressions for the multi-ion potentials. Evaluation of these potentials as well as utilizing of them in metal property investigations are highly desirable to provide a convincing verification of the approach proposed. It will be a subject of the next paper.

\section{References}

[1] I.R. Yukhnovskii, Z.A. Gurskii, Quantum-Statistical Theory of Disordered Systems, Naukova Dumka, Kiev 1991, p. 287 (in Russian).

[2] F. Ducastelle, Cohesion and Structure Order and Phase Stability in Alloys, North-Holland, Amsterdam 1991, p. 511.

[3] J. Hafner, From Hamiltonians to Phase Diagrams, Springer Verlag, Berlin 1987, p. 404.

[4] M.P. Allen, D.I. Tildesley, Computer Simulation of Liquids, Clarendon Press, Oxford 1987 , p. 400.

[5] H. Böttger, Principles of the Theory of Lattice Dynamics, Academic-Verlag, Berlin 1983 , p. 382.

[6] V.G. Baryakhtar, E.V. Zarochentsev, E.P. Troitskaya, Computational Physics Methods in Solid State Theory. Atomic Properties of Metals, Naukova Dumka, Kiev 1990, p. 373 (in Russian).

[7] J.A. Moriarty, Phys. Rev. B 38, 3199 (1988).

[8] W.A. Harrison, Pseudopotentials in the Theory of Metals, W.A. Benjamin, New York 1966, p. 366. 
[9] J.A. Moriarty, Phys. Rev. B 16, 2537 (1977); 26 1754, 82 (19.)

[10] S.H. Wej, L.G. Ferreira, A. Zunger, Phys. Rev. B 41, 8240 (1990).

[11] B.L. Gyorffy, G.M. Stocks, Phys. Rev. Lett. 50, 374 (1983).

[12] P. Turchi, G.M. Stocks, W.H. Butler, D.M. Nicholson, A. Gonis, Phys. Rev. B 37, 5982 (1988).

[13] A. Pasturel, V. Drchal, J. Kudrnovsky, P. Weinberger, Phys. Rev. B 48, 2704 (1993).

[14] J.W. Connolly, A.R. Williams, Phys. Rev. B 27, 5169 (1983).

[15] Z. Gurskii, J. Krawczyk, Metal Physics and Adv. Technologies 18, 3 (1996) (in Russian).

[16] P. Hohenberg, W. Kohn, Phys. Rev. B 136, 864 (1964).

[17] W. Kohn, L.J. Sham, Phys. Rev. A 140, 1133 (1965).

[18] V.V. Nemoshkalenko, V.N. Antonov, Computational Physics Methods in Solid State Theory. Band Structure Theory of Metals, Naukova Dumka, Kiev 1985, p. 407 (in Russian).

[19] M.C. Payne, M.P. Teter, D.C. Allan, T.A. Arias, J.D. Joannopoulos, Rev. Mod. Phys. 64, 1045 (1992).

[20] S.H. Vosko, L. Wilk, M. Nusair, Can. J. Phys. 58, 1200 (1980).

[21] J.P. Perdew, A. Zunger, Phys. Rev. B 23, 5048 (1981).

[22] Theory of Phases in Alloys, Ed. A. Katsnelson, Nauka, Novosibirsk 1984, p. 223 (in Russian).

[23] L. Kleinman, D.M. Bylander, Phys. Rev. Lett. 48, 1425 (1982). 